\title{
OpenCare Project: An Open, Flexible and Easily Extendible Infrastructure for Pervasive Healthcare Assisted Living Solutions
}

\author{
Stefan Wagner \\ Engineering College of Aarhus \\ Computer Technology and Embedded Systems \\ Aarhus, Denmark \\ sw@iha.dk
}

\author{
Claus Nielsen \\ University of Aarhus \\ Department of Computer Science \\ Aarhus, Denmark \\ cln@cs.au.dk
}

\begin{abstract}
Pervasive healthcare technology is needed to meet the challenges of an aging population in the western world, as well as an expected major shortage of healthcare personnel in the near future. The OpenCare Project is an open source infrastructure framework, designed to be used by researchers and corporate developers for developing experimental assisted living prototypes, as well as a complete feature rich systems with a high degree of flexibility and modifiability. Following a motivating discussion on the problem domain, a survey of related research projects and commercial products, points out the need for an open source infrastructure framework. Finally, the OpenCare Project is presented and the overall design is discussed.
\end{abstract}

Keywords-component; pervasive healthcare, pervasive computing, ubiquitous computing, home care, assisted living, infrastructure, sensor networks, vital signs, sensor, NFC

\section{INTRODUCTION}

This paper discusses the pervasive healthcare-based OpenCare Project and Infrastructure. An open source infrastructure project and framework for implementing and testing assisted living (see section III) prototypes and systems, connecting various vendor's sensor hardware and software components with end-users, caretakers, administrative staff, relatives, utilizing alerts and notifications through different media, SMS \& MMS text, email, messaging, web, databases, web services, electronic patient records and more.

In short, this framework targets research groups and commercial companies and public institutions, wishing to implement their tailor-made assisted living solutions on an open source platform and framework, without getting locked to a specific vendor or platform, and being able to use a range of existing commercial sensors, along with their own custom-made hardware and software components, and running on different hardware and operating system platforms.

We start with a discussion on the background and motivation for utilizing pervasive healthcare technology and assisted living technology, in particular looking at facts and figures on the demographic development in the developed countries, which is considered as becoming increasingly problematic in the not too distant future by many researchers. We will argue that pervasive technology might be used to overcome some of the economic and staff shortage problems facing the healthcare sector as a consequence of these demographic projections. Then, the field of pervasive healthcare and assisted living is introduced, along with the pervasive and ubiquitous computing paradigms.

A survey of related research and commercial systems in the area is given, analyzing on the features, strengths and weaknesses of these systems, followed by a discussion for the need of an open, flexible and easily extendible infrastructure, as an alternative, or supplement, to these systems, in particular for other research projects. Finally a short introduction to some of the components of the OpenCare Project and Infrastructure is given.

The framework is under ongoing construction, but a fully functional system is running and available for interested partners, free of charge, and with the full code base. We have already added driver support for several different commercial state of the art sensor products, including ECG, HRV, blood pressure, blood sugar, pulse and oximeter, weight and other sensor types. Also, several research projects are working on implementing driver support for their hardware units, including a Heart Rate Variability sensor project and the Automatic Medicine Dispenser project. 


\section{BACKGROUND AND MOTIVATION}

The western world is facing a major shift in its demographic composition, and this constitutes a potential threat to the economy of the existing healthcare systems. This change is a result of many factors all related to the increased wealth and social security in the industrialized world which have been established in modern time. This increased wealth has lead to major advances in sanitation and other public health related engineering areas, access to an abundance of healthy food and clean drinking water, and not the least in broad public access to ever better and more efficient and relevant medical treatment, including a wide range of pharmaceuticals counting amongst others a range of different antibiotics and vaccines, as well as advances in medical technology and surgery. These advances and others have resulted in a dramatic increase in the overall expected lifespan of a western world citizen, allowing more children to survive to become adults, and allowing adults to live far longer lives than previous generations.

On Figure 1. to Figure 4. demographic data and data projections are shown from two very different but yet comparable western world countries, the United States of America with a partly private healthcare insurance based system, and Denmark with an almost exclusively publicly funded healthcare system. Denmark with a total population of 5.3 million with an average life expectancy of 76.7 and the US with a total population of 282.3 million in the year 2000 , and an average life expectancy of 76.6 years. These figures should be compared to a world average of 63.8 years and 62.4 in the group of less developed countries [1]. At the same time, in 2000 the US spent $13.3 \%$ ( $16 \%$ in 2005$)$, of its gross domestic product per capita on health expenditures, or $\$ 4,588$ USD compared to Denmark spending just $8.3 \%$ or $\$ 2.555$ USD per capita, and with the same life expectancy as a result. For a comparison, Turkey had a mere $\$ 459$ USD per capita in 2000 , which still constitutes $7.4 \%$ of their gross national product.

The U.S. Census Bureau has collected projections on the demographic composition of the future. According to their figures, the percentage of the population in the US over 65 years will rise from $12.4 \%$ in 2000 , to $18.2 \%$ in 2025 and to $20.7 \%$ in 2050 - or almost a doubling in 50 years. The same numbers in Denmark are $14.8 \%$ in $2000,21.2 \%$ in 2025 and $24.7 \%$ in 2050 , or roughly the same percentage increase as in the US.

The percentage of the population becoming older than 80 years will in the US increase from $3.3 \%$ in 2000 , to $4.5 \%$ in 2025 , to $8 \%$ in 2050 . The equivalent Danish numbers are $4 \%$ in $2000,5.9 \%$ in 2025 and a staggering $9.7 \%$ in 2050 . For both countries this amounts to an estimated increase of almost 2.5 times as many citizens at or above the age of 80 .

Heart and circulatory conditions increase from being $0.53 \%$ at the ages $18-44$, to $2.53 \%$ ages $45-54$, to $6.6 \%$ in age $55-64$, climbing to $9.67 \%$ for ages $65-74,15.35 \%$ ages $75-84$ and $22.07 \%$ by 85 and over. This shows a significant rise in the group of people over age 64 .

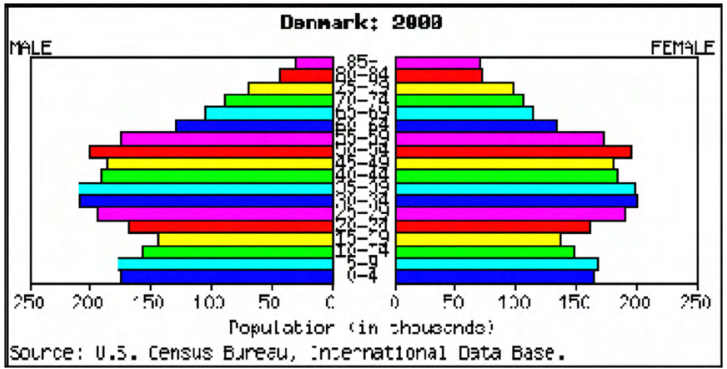

Figure 1. Distribution of Danish population of age and gender 2000 Source U.S. Census Bureau, International Database[1]

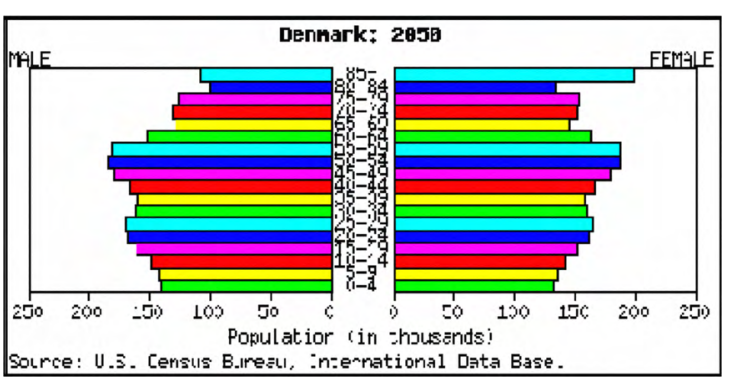

Figure 2. Distribution of Danish. population of age and gender 2050 Source U.S. Census Bureau, International Database[1]

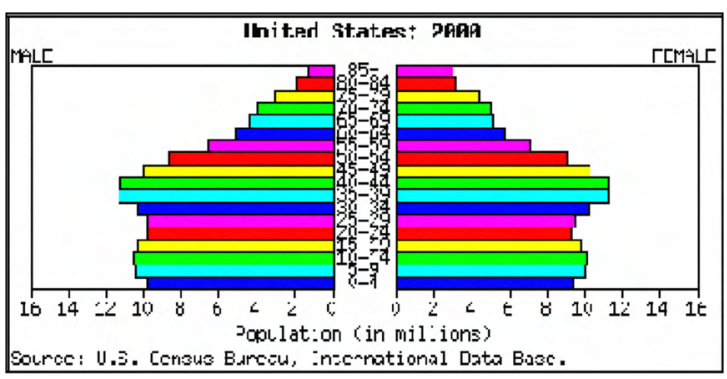

Figure 3. Distribution of U.S. population of age and gender 2000. Source U.S. Census Bureau, International Database[1].

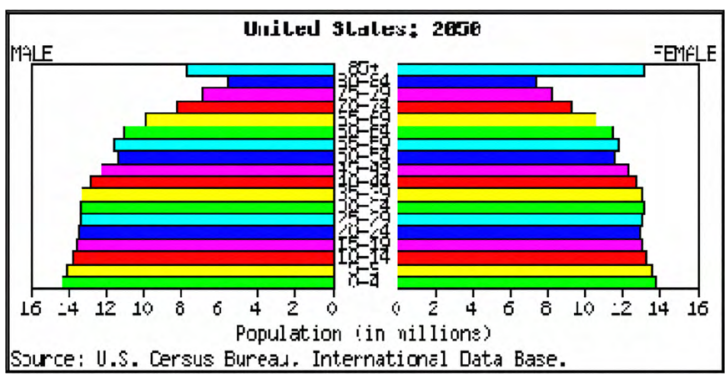

Figure 4. Distribution of U.S. population of age and gender 2050. Source U.S. Census Bureau, International Database[1].

The same tendency is seen with other chronic conditions, for instance is non-insulin requiring Diabetes (type 2) 
increasing from $1.32 \%$ in the ages $45-54$ to $4.06 \%$ by ages $65-74$, or almost a four time higher incident rate in a 20 year interval only.

Americans over age 65 thus incur considerable higher medicine expenses than their younger countrymen, requiring more caretaking staff, equipment and facilities [2], [3]. It is estimated that over 600 million people worldwide have chronic diseases, and the spending on chronic diseases is expected to increase [4]. In the US alone, spending is expected to increase from $\$ 500$ billions a year to $\$ 685$ billions by 2020 [4].

At the same time that the number of elderly citizens requiring a higher degree of caretaking and service is growing rapidly throughout 2000 to 2050 , the number of people traditionally considered within the working-age, that is, below 64 or 70 depending on culture and regulation in individual countries, is decreasing accordingly. In the US, in the year 2000 there was approximately $59 \%$ of the population within the working-age of $20-64$, and only $12.4 \%$ in the older adult group, which is not considered part of the working force by today's norms. This is around 4.7 working men and women for every retired or elderly adult. By 2025 this will have changed to 55.4 vs. 18.2 or 3 workers for each elderly. And in 2050 we end up at 53.5 vs. 20.7 - or merely 2.6 active workers for each elderly. The same ratio numbers for Denmark today (2000) are 4.2, and 2.7 for 2025, and as low as 2.2 active workers for each Danish elderly by 2050 . This is a half of the workforce compared with the year 2000 , and they will thus be facing at least twice the workload for each caretaking professional by the year 2050 .

We may increase the potential active work force available by different means and incentives. This includes importing young and educated caretaking workers from third world countries, which do not suffer from the same bleak demographic scenarios. Or we may offer incentives for caretaking staff to work more hours, shortening duration of the healthcare educations and finally by keeping healthcare workers longer on the job market through incentive programs and other. Also, the workers may increasingly come from other sectors, as these no longer requires the same number of workers (for example from the heavy industry, and other industries being increasingly automated or outsourced).

These issues are, however, not investigated further in this paper, although they are of course most relevant as alternatives or supplement to a solution focusing on technical advances.

The other path suggested in this paper, is through the use of technology in order to increase the efficiency of the limited staff resources. This process is not in any way new, and the use of computers and electronic medical equipment has long been a major growth area.

The new paradigm within healthcare technology has been dubbed pervasive healthcare [7] (see section III on pervasive healthcare) and hails a new approach to supporting caretaking staff, patients and relatives. The area includes both the primary and the secondary healthcare sector.

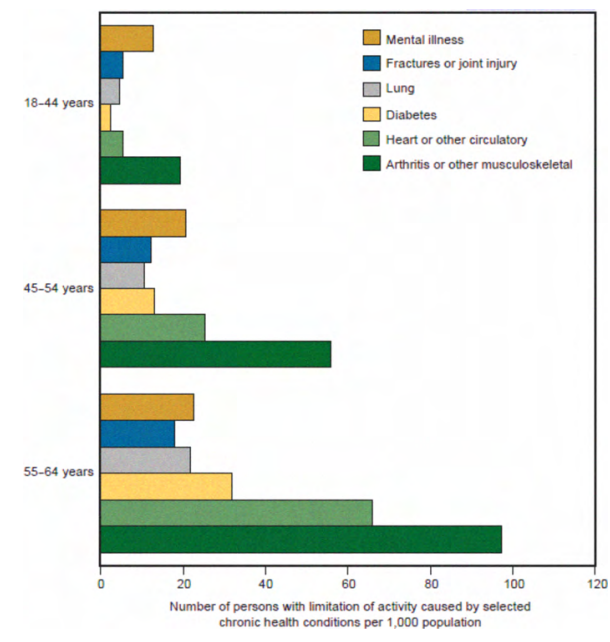

Figure 5. Limittation of acitivity caused by selected chronic health conditions among working-age adults by age: United States, 2004-2005 [5]

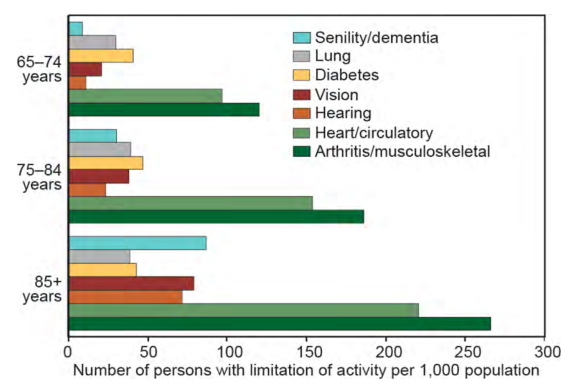

Figure 6. Limittation of acitivity caused by selected chronic health conditions among older adults, by age: United States, 2004-2005. [6].

The primary sector consisting of home and communitybased healthcare, including the home nurses, nursing homes and general practitioners, while the secondary sector mainly consists of small to large seized hospitals (depending on the healthcare organization of the individual country or region). The secondary sector receives a great deal of attention, and it might appear to be the most relevant sector to focus on, trying to achieve a heightening of efficiency here. It is however very likely that focusing the effort on the primary sector, by helping people in their own homes, including monitoring their condition (ECG, heart rate, blood sugar etc), and making sure they take their prescribed medication, might prevent them from ever being admitted to a hospital, and as such it will decrease the expenses incurred of a hospitalization.

As suggested by [8] the price for a hospital bed for one day amounts to a mean of $\$ 1,237$ USD, varying between $\$ 2,401$ USD for critical or intensive care days, to $\$ 1,122$ USD for regular care (2005 numbers for the state of Michigan). The estimated average cost in a Danish public sector hospital is around $\$ 1,104$ USD per day [9]. 
Of course, these figures may vary to a large degree between different healthcare systems, depending on wages and level of facilities, but they do indicate the overall level of the cost of being hospitalized. If we can avoid just a few days of hospitalization by increasing the quality of home care, we may achieve significant savings for each day of hospitalization avoided. This could easily finance the technology needed, while at the same time save the expected future scarce manpower available.

Also, the vast majority of Americans over the age of 65 live independently in single family homes [10] and [11]. They spend a major part of their time in the home, including around 6 hours spent sleeping [11]. It is thus most likely, that it is in the home where the realization that someone is sick first occurs, as well as where the elderly feels the most at home and confident. The earlier we might be able to help the occupant understand his current condition and what to do next, the easier it will be to keep him out of hospital.

The OpenCare Project is primarily focusing on the primary sector, the home and community-based health area. Of course, hospital staff may also use some of the information from the system, but the main objectives include early warning, information, reminders, help and guidance, as well as collecting data for better decision making for the caretaking staff, the elderly themselves and their relatives.

\section{ENABLING TECHNOLOGIES}

\section{A. Introducing the Main Theoretical Paradigms}

In the following sections, we will discuss the main theoretical paradigms, frameworks and technologies that we later on will argue might help in obtaining the increased efficiency to ease the demographic related problems we are facing as discussed in the previous section.

We start from the top of the theoretical pyramid, introducing the pervasive computing paradigm, which is more of a way of thinking interaction and computer support than it is a well-defined computer science area. We then take on the concept of pervasive healthcare, which might be considered a subset of pervasive computing within the healthcare sector. Finally we will look at assisted living, which is pervasive healthcare at work for the end-users (in some cases considered patients) in their homes.

\section{B. Pervasive Computing}

The concept of pervasive computing, or ubiquitous computing as it was originally coined, may be attributed to the researcher Mark Weiser. Weiser [13] was a zealous advocate for finding alternative solutions to the $\mathrm{PC}$, which he deemed to be counter productive to getting more technology support integrated into people's daily lives. Indeed, Weiser envisioned the proliferation of technology throughout the workplace to allow for tighter integration with the actual work tasks. Amongst the technology solutions Weiser and his colleges produced for supporting this vision, was the concept of "Tabs, Pads \& Boards" devices, communicating with each other through a wireless network, and the ability to track the location of a user within his ubiquitous equipped laboratory and support work tasks in novel ways.

This technology vision has now become a commercial reality. PDA's and cell phones, able to run applications and communicating using WiFi or mobile networks such as the Global System for Mobile communications (GSM) or Universal Mobile Telephone System (UMTS), are now available at a cost that may be considered negligible by most in the industrialized world.

Weiser's main vision was that of "calm technology". Letting the technology disappear from our attention, and instead let the technology help us, rather than allow it to be in our way. A design goal also discussed by Norman in his book on "the invisible computer" [15].

Much of the current research literature on pervasive and ubiquitous computing in general, and pervasive healthcare in particular (which we shall cover in the next section), considers the mobile phone to be the essence of Mark Weiser's vision, solving all the pervasive tasks we might imagine, including healthcare related tasks. We tend to disagree with those people, however. To Mark Weiser, the notion of tabs (which has some resemblance with the mobile phone of today), pads and boards, was only the "ends to a mean". As Norman stated, a device to "rule them all" - or a device that handles everything, being both a phone, a walkman, a calendar, a camera and a healthcare hub, is not a usable product - or at least not a product usable by all. And if we consider some of our target groups for this project, namely the elderly (and perhaps) cognitive impaired, one should be very careful to be considering the mobile phone as the perfect ubiquitous device for solving pervasive computing tasks. Most commercially available mobile phones are not in any way a "calm" experience for many elderly users, and in fact, applications for mobile phones does often not work as easy and painlessly as expected.

So, pervasive computing technology implies many other things than just being mobile and being able to access data anywhere, anytime. It is also about creating contextual awareness, and about being proactive, and perhaps more important, about being user friendly to the extreme. If we need to harness the power of technology out in the homes of potentially cognitive and physically impaired people, we must exploit the full potential of Weisers original ideas. Sensors must be aware where the user is, what the user is doing, and perhaps what the user should be doing. If he for instance is in the bathroom, and he has not taken his medicine as prescribed, the system should be aware of his position, and be able to provide him the info in the context he is in. It is no use that his phone is vibrating to remind him to take his medicine, if he is sitting in his bathtub.

Pervasive computing is about sensing and intelligent decision making. Contextual awareness and of course, access to data and the flow of data. If the elderly patient (from before) did not take his medicine, then someone should be informed of it. It might be his doctor - which he will see next month - or it might be a simple text SMS message sent to one of his relatives, friends or spouse. 
Pervasive computing enabling technologies include sensors and actuators, regular PC's, embedded computers and mobile devices. The use of wireless technologies is of major importance in pervasive computing. Technologies include WiFi, Bluetooth, ZigBee, RFID, NFC, TCP/IP, while sensors might be everything from accelerometers or visual movement trackers to measure movement, heat sensors, pressure sensors and more. Speech and voice recognition technology certainly also goes under the notion of pervasive enabling technology, allowing for a calmer and more natural user interface - as is the use of touch screens and other direct manipulation interfaces.

\section{Pervasive Healthcare}

Pervasive Healthcare is healthcare technology within the pervasive computing paradigm. That is - instead of thinking healthcare technology as following the traditional personal computing paradigm, we apply the special techniques and technologies that are featured by pervasive computing theory.

As an example, the use of a home blood pressure measuring device might be considered "pervasive" by some, as it allows the user to sit at home, to measure his blood pressure, and either record the data in a booklet, phone the doctor or nurse, or enter the data in an email or even a PC application. We do not recognize this to be an example of pervasive technology neither in theory nor applied. If the user instead performs the measurements, and does not have to worry about the data getting to the doctor and in his medical records, as this happens automatically, this would be one step closer to a pervasive computing scenario. In fact - if the user had to do absolutely nothing, where he needs to worry about installing the infrastructure and getting the data sent, then that is even closer to "calm technology". Most calm or pervasive would it be, if the user was not even aware that he was being monitored (of course after having given his permission), then that would be truly calm technology.

Our definition of what is indeed pervasive technology and what is not, is quite perilous. What might be considered pervasive by some might not be appreciated as such by others. The field of pervasive healthcare is not sufficiently rigidly defined to truly deal with this, as is neither pervasive computing. But again, falling back to Mark Weiser's teachings, pervasive technology should be as calm and invisible as possible.

Bardram et al. [7] give the, in their own term, superficial definition of "pervasive healthcare" as being the application of pervasive computing in healthcare. They do however propose, that pervasive healthcare, like pervasive computing, is a truly multidisciplinary field, involving many different technologies and schools of thought.

Bardram et al. also recognize the different aspects of pervasive healthcare whether in a hospital setting, or in the home of the patients or end-users.

\section{Assisted Living}

Pervasive healthcare at the user's home is sometimes called assisted living or assisted cognition [16]

These terms have been used to describe systems that use sensor data to determine what current activities the user is doing while at home, and guide the user in order to solve the task more easily or safely, as well as monitor the person's health condition. This might include reminding the user to take his medicine as prescribed, warn about a potentially too high blood pressure, or an arrhythmia of his heart - advising him to rush to the hospital or seek help.

Assisted living is really about making life easier and safer for the users and their relatives - and for extending the tools available to home nurses, general practitioners and perhaps hospitals staff or other specialist functions.

For instance, a patient who is suffering from Meniere's disease (a build-up of fluid in the inner ear) as well as having increased blood pressure, might be dependent on taking her medicine at a regular interval in order not to get dizzy and fall (perhaps breaking her hip or other bones in the process) or risking a heart attack, heart failure or stroke. An assisted living system might remind her to take her different types of medication, using visual or audio signalling, or even just telling her using speech technology. The system might have sensors able to detect her leaving her home, so that she might be reminded of taking the medicine before she leaves. Same system might also ask her to take her blood pressure at regular intervals (once or three times a day), and automatically store the data and send them to the physician she is attending for next check-up. He would be able to watch reports on how often she forgets taking her medicine, and what effect it has on her blood pressure when she does not remember to take her medication.

If we now also equip her with an accelerometer unit, we might measure her level of activity, including amount of sleep and rest. These data again goes to the physician for the monthly check-up. Same accelerometer unit might be able to detect if she falls, and stays down in case of e.g. a broken hip. And now - the system might send an SMS text to one of her relatives, or to a private surveillance company or the local community nursing facility, depending on resources available.

This vision of an assisted living system is exactly the type of system that the OpenCare Project supports and facilitates. Including the OpenCare Infrastructure that makes it easy to attach the sensors, the drivers developed for the blood pressure meter and the automatic medicine dispenser. Other supported features include the messaging and alert API for sending data to the physician and relatives and many other that will be discussed in section VI.

The OpenCare Project and Infrastructure is not a complete system for assisted living projects. It is an infrastructure which is designed to be as open and flexible as possible. It is an infrastructure for other researchers and commercial vendors to design and test prototypes, and eventually perhaps developed complete systems based on it. 
Many of the ideas of the OpenCare Project are not original, and have been discussed in earlier literature. There are also some commercial systems available for assisted living, so the OpenCare Project is not necessarily a revolution. There are several new ideas behind the OpenCare Project, but the most important being the open source nature of the project.

In the following we will analyse related research projects and commercial products, and discuss the features, strengths and weaknesses of these, and discuss where the OpenCare Project might be better suited or not to solve the same problems.

\section{RELATED RESEARCH AND COMMERCIAL PRODUCTS}

It will not be possible to cover all research projects within the field of assisted living nor all available commercial products on the market. Furthermore - we will focus on infrastructure projects rather than for example pure sensor projects or usability studies, limiting the candidates further.

Seon-Woo et al. [17], are looking into the assisted living domain surveying the elderly in their own homes. An architecture is created with sensors for detecting movement and a gateway in the home for sending the data to a central server. They focus primarily on analysing the data. The system appears to be rather monolithic by nature, and does not seem open. This might be a good strategy to solve the particular scenario they are aiming at and have set as their objective, but what if the system is later adapted by a regional care center and the need arises for the system to talk with several back-end systems, or they need to support a range of hardware sensors from another vendor. Whether there is access to the source code and whether this is open source is not discussed, but this appears not to be the case, and thus not allowing for modifications or adaptations of the system.

Huang and $\mathrm{Su}$ [18] also looks into establishing an architecture for the home, but focuses on the conversion of signals in the home to a known format. The converted data is placed in a middleware where the format from e.g. a webcam or an ECG is formatted to the DICOM [19] format from NEMA [20]. Due to the limited amount of information on the middleware in relation to the use of technologies, this work is again deemed rather closed and limited on features. The use of standards (DICOM and NEMA) seems quite relevant, and using a middleware could be promising, if the middleware was itself heterogeneous with regards to programming languages and operation system platforms. This is not possible to deduct from the text, as is neither code availability nor licensing issues.

Varshney [21] writes about the infrastructure for monitoring the elderly at home. The research focuses on how sensors should communicate with the outside world, but does not specify a complete solution. This is again deemed a prototype project - exploring a single case, rather than providing a complete infrastructure for assisted living solutions.
Bambis et al. [22] discusses the possibility of monitoring the elderly in their homes and tracking their movement and providing a scalable framework for interpreting their movement data and a state model of their behaviour. This includes an API for asking whether the person is getting enough sleep, where the person currently is, number of visits to the toilet etc. The system allows for third party movement and tracking sensors, but it does not include e.g. ECG or blood pressure sensors. The system appears modularized and scalable. It does not however appear to be open for third party software systems, as it does not appear to be available for source code download. The system share many similarities with the OpenCare Project discussed in this paper, but appear as being rather monolithic and closed.

Kirovski et al. [23] from Microsoft Research introduces the Health-OS system. It is a system, apparently dedicated for the Microsoft Windows and Windows CE platforms (even though it is claimed it will run on any suitable real time OS based device), which integrates different commercial sensor types, collects and stores the data on standard Windows and Windows CE devices. The system appears somewhat open, including an SDK for accessing the data and functionality. However the source code does not appear to be available, again keeping the system closed for most extensions or modifications.

Schwiebert et al. [24] discusses some issues regarding biometric sensors where they point out a number of important points including problems with power supply, interruption of the elderly etc. The project is deemed more relevant for providing requirements for sensor manufacturers than it is relevant to infrastructure discussions.

Rodrig and LaMarca [25], presents a P2P architecture for collecting and distributing data from sensors. In our opinion, the complexity of an infrastructure tends to increase when using a P2P architecture, and Rodrig and LaMarca does not convince us otherwise with their system. This does not mean that it might not have its validity in some scenarios, just that a pervasive healthcare infrastructure not seems to gain anything from being P2P based.

Bardram et al. [26] discusses the transition from ordinary visits at a physician to the use of telemedicine, where the patients themselves performs the measurements and collects the data. The paper does not focus on infrastructure or architecture, but on the usage of systems suitable for home use.

Telcomed [27] is a commercial company based in Ireland, producing a range of telemedicine and pervasive healthcare products. Including sensors for monitoring heart rate, 1 lead ECG, body temperature, weight, blood pressure, blood sugar and more. They provide a complete infrastructure, from the patients home to the web-based Telcomed monitoring center. They use proprietary communication protocols, and the solution appears not to be open for third party solutions.

Corscience [28] is likewise a commercial company, based in Germany. They also provide a complete vertical 
monolithic solution including sensors for ECG, blood pressure (both 1-lead and 3, 6 or 12-lead), a weigth sensor as well as an Asthma sensor device. The Corscience system can send data from either a stationary gateway, or a small mobile gateway, to a central database, and provides web-access to these data, including alert functionality and more. The Corscience system is closed like Telcomed, but they do however use Bluetooth for most sensor devices, and they do share their communication protocol with partner companies, signing an NDA. The OpenCare Project is supporting several Corscience sensors devices at present, incl. ECG, blood pressure and weight. Corscience is not open source, and not much more open than is the Telcomed solution. Again, choosing Corscience requires a full solution, and it may not work with e.g. Telcomd products.

Yet another private company is Tunstall. Tunstall [29] has a wide range of sensor products, incl. pressure mats and movement sensors, as well as blood pressure, ECG, temperature and more. Tunstall utilizes an interactive basestation at the users home, sending the data directly to the relevant clinic. Again, the sensors are Bluetooth based, and by signing an NDA, it is possible to partner with them. Again, we see a semi open solution, which is however not open source.

Intel has recently released the Intel Health Guide PHS6000 [30], which is an interactive mobile computer with a touch screen, camera and more. According to Intel, a whole range of sensor types has been certified for use with it, which sensors exactly has however not been revealed yet. The solution appears somewhat monolithic in nature, but might be open for third party sensors, as long as Intel will certify them. There is no mention of whether the system is open for external systems, including Electronic Patient Record systems, clinical systems and others. Also, there is no mention of whether the source code is available for modifications, or whether the hardware platform might be changed to something else than an Intel based machine.

The Continua Alliance [31] is a non-profit, open industry alliance of healthcare and technology companies that has realized that "broad interoperability has yet to be achieved" [32] within the field of pervasive healthcare. They conclude that "Much of the technology that can improve healthcare already exists in some form. For example, medical devices that monitor health and fitness - blood pressure cuffs, glucose meters, medication trackers, weight scales and pedometers - are on the market; however, these pieces cannot be integrated into full personal telehealth systems that can send data from multiple vendors' medical devices to a health care provider or fitness coach. No standards exist that fully define interoperability among these devices, thus the market is unable to invest in interoperable solutions." [33].

The Continua Alliance has the goal of achieving interoperability between different healthcare related technologies and solutions. The Continua Alliance plan on using the ISO/IEEE 11073 family of Medical Device Connectivity standards and Bluetooth and the USB Personal Healthcare Device Class Specification as the glue to tie healthcare sensors and computing hardware together with Remote Patient Monitoring and Electronic Health Record systems [34]. The Continua Alliance is dedicated to provide developer resources (including access to some free reference source code) as well as guidelines and a certification process. The Continua Alliance is as such in many respects closely related to the mission of the OpenCare Project. However, they are more concerned with device interoperability than providing a complete and freely available end-to-end infrastructure.

\section{LESSONS LEARNED FROM RELATED RESEARCH AND PRODUCTS}

Most existing research projects and commercial products on the marked, as discussed in the preceding chapter, uses closed proprietary software and hardware solutions, not allowing individual integrators to add third party sensors or requiring a certification process. Only to some degree do such products provide third party software access, e.g. with limited web service or other middleware support to some of the data. Furthermore none of the related research products and commercial products offers the flexibility of providing access to the source code for extension or modification.

An optimal infrastructure-architecture should allow caretaking professionals, institutions, companies or individuals, to freely choose among the most appropriate sensor types and software components suiting exactly their needs, thus avoiding the inherent risk of vendor lock-in ${ }^{1}$ and basing a pervasive healthcare infrastructure for home care and assisted living systems on a non-extendable and inflexible platform.

Much published research within the pervasive healthcare field, including the related research presented in the above, are to a large extend not supporting this. Many research projects choose to build their own infrastructure to deploy projects within. Producing a robust, efficient and safe tailormade infrastructure for a research project is highly resource consuming, and will most often not be able to provide the same features and security mechanisms than a general purpose healthcare infrastructure would. We may only speculate why so many research projects chooses to build their own infrastructure, but one possible reason is the pricing of existing commercial infrastructures, and the fact, that most of these are not open for extension, or even modification of the existing code base. Systems should be open and flexible with regards to potential modification - so that researchers and corporate developers can easily adapt the system to specific needs in order to test new sensor types - or experiment with new ways of using existing technology.

True openness and flexibility may only be achieved by having an open source framework, where you might freely

\footnotetext{
1 "Vendor lock-in, or just lock-in, is the situation in which customers are dependent on a single manufacturer or supplier for some product, or products, and cannot move to another vendor without substantial costs and/or inconvenience."
} 
modify the existing code. For every new participant project, the code base may be increased with new features.

The OpenCare Project and Infrastructure is exactly this. An open source infrastructure for pervasive healthcare projects with focus on assisted living solutions. The OpenCare project aims at providing a framework for producing tailor-made infrastructures, while also providing drivers for many commercial sensor types, and examples of usage. In the following we will provide an overview of the project.

\section{OVERVIEW OF THE OPENCARE PROJECT}

The OpenCare Project is divided into four logic tiers. The Home, Central, Public and Mobile-tier. The Home-tier logically belongs at the end-users home, while Central-tier contains the central server and database, as well as various business-logic code.

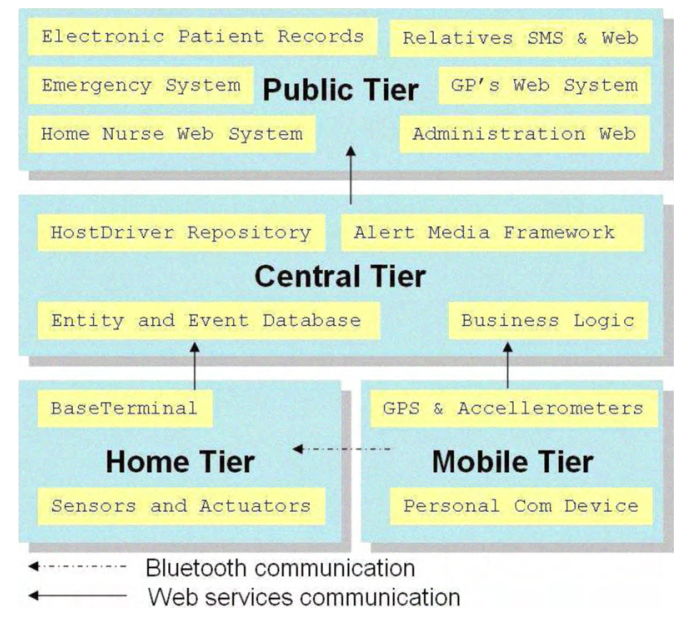

Figure 7. The four tiers of the OpenCare Infrastructure, along with the major components contained within them.

The Public-tier constitutes the facades for the administrative staff and professional caretakers, hospital staff, general practitioners, and home nurses and other caretaking staff. Represented by either a PC, web or mobile solution. Also, the Public-tier is available as distribution services, including as SOA-based ${ }^{2}$ web services and other supported heterogenic distribution middleware standards.

The Home-tier consists of a stationary touch screen unit in the end-users home (but may also be a regular PC or even a headless unit), including a near-zero-configuration framework [37], a reminder framework, a persistency framework and an alert and alert media framework. Also, a symbiotic mobile framework (OpenCare Personal Communication Device) is under development, allowing the user the freedom of leaving his or her home while continuing being monitored - shifting to the Mobile-tier.

\footnotetext{
${ }^{2} \mathrm{SOA}:$ Service Oriented Architecture
}

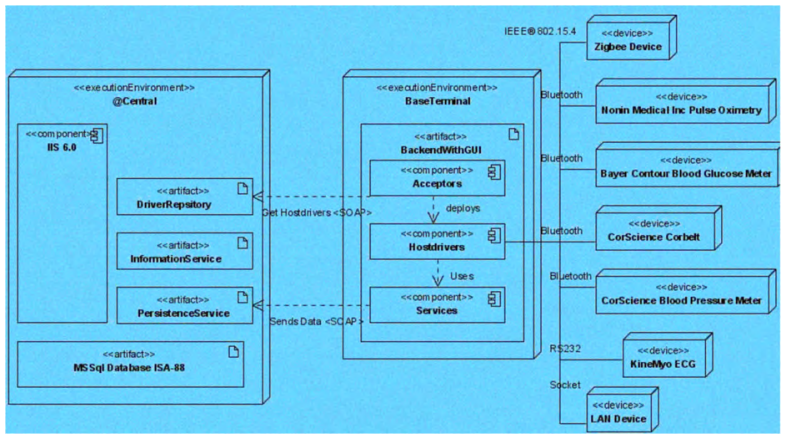

Figure 8. Diagram illustrating how a potential deployment, using the OpenCare Infrastructure framework, could be realized. This diagrams shows two separate execution environments/systems; The Central-tier and Home-tier/BaseTerminal. Both systems utilizes the OpenCare Framework.

It has previously been argued that usability and efficiency are some of the primary success criteria in order to get a pervasive healthcare system successfully deployed in the user's homes [36]. We cannot expect the relatives or caretaking staff who are installing and handling these systems, or new sensors, to have the necessary technical expertise to handle complicated installation procedures.

Therefore all installation should occur with zero or minimal configuration (near-zero) efforts. To ensure this, an essential part of the system is the near-zero-configuration sensor framework [38], which allows sensors and base stations to configure and pair automatically. This is accomplished through Bluetooth, WiFi and other wireless and wired technologies as discussed in [37]. Minimal required user interaction is one of the most important elements of ubiquitous and pervasive computing, and this is thus a very important aspect of the system, and is widely implemented at current.

As stated earlier, the system today supports a wide range of wireless sensor types from different manufactures, and all of these may be automatically detected, which will lead to automatic driver download and activation in the running system in the users home, without any kind of user interaction. As such the system achieves a kind of zeroconfiguration, or almost zero-configuration, as the use of a $\mathrm{NFC}^{3}$-device-based binding mechanism is necessary, as true zero-configuration has proven not to be practical possible to implement, as discussed in [37]. While there are no sensor range overlap issues in isolated installations, the originally constructed framework architecture proved to suffer in the case of several patients being within range of each others base stations. The zero-configuration units are unable to determine which sensor belongs to which patient and base station, in case of these not being either preconfigured or configured by manual onsite user interaction, thus breaking the zero-configuration paradigm. This problem has been solved by using NFC-enabled phones, and NFC-labels and smart cards.

\footnotetext{
${ }^{3}$ NFC: Near Field Communication
} 


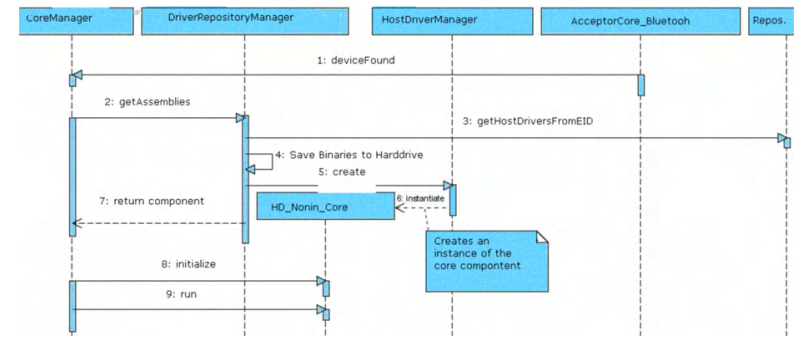

Figure 9. Sequence diagram illustrating how hostdrivers are acquired. A Bluetooth Acceptor has discovered a new Bluetooth device, and attempts to download a hostdriver from the central driver repository based on an ID (UID, which here is the MAC-adress of the device). Note that only the UID is used to acquire the hostdrivers from the DriverRepository. Different acceptors exists for different communication technologies - including one for Bluetooth, one for serial, one for ZigBee, one for uPnP, and new acceptors may be deployed dynamically.

In the Central-tier we find the domain model of the system, modelling users, equipment, relations (including who should receive which data, in which formats and on which media). For instance, the users being monitored are found in this database, including personal records, a reminder calendar, the data that has been gathered from sensors and other sources, as well as which caretakers and relatives should receive status information and data from them, including alerts on media such as SMS texts, email, callbacks to surveillance software and more. Also in the Central-tier, we find a service layer, servicing the Home-tier, and thus the software running in the user's homes. There is also a layer servicing the Public-tier. A range of different heterogenic distribution middleware types are supported here, including web services for maximum openness to different operating system and programming language platforms. In the Centraltier, the hostDriverRepository is also hosted, which contains all known drivers for any sensor devices supported by the system. Usually a device is identified using its MAC-address or its Service signature (which is the case for most Bluetooth-based devices).

New hostdrivers must implement the IHostDriver interface, which must then be compiled to a .NET assembly and deployed on the repository, and the database needs to be updated with its UID. This is all that is needed to add a new hostdriver to a running system, and start deploying software.

The Public-tier is divided into several views providing different functionality for different user groups. One view targets the administrative staff, providing features for creating the data entities: users, sensor types, host drivers etc. This is provided by a web application for easy deployment. However, in order to allow maximum flexibility and openness, all functionality is also supported by a distribution middleware, so that it is easy to integrate the administrative functionality in existing applications, including PC, UNIX, LINUX, Apple or web based, not locking developers to the web platform. Again, as all code is open source, one can easily change the code as preferred, while the modularized design further enhances this

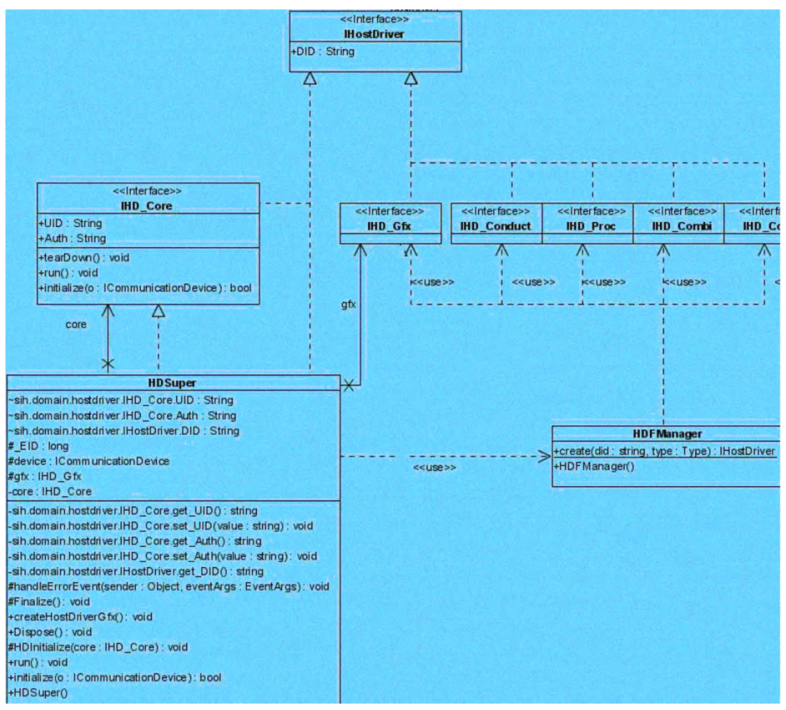

Figure 10. Class diagram showing the content of the sih.domain.hostdriver subpackage. The term "Hostdriver" relates to a collection of components together representing the concept of a hostdriver. All the hostdriver component inherit the IHostDriver interface which helps to identify the

hostdriver and decouples different hostdriver assemblies and objects.

The Public-tier also provides a view for the associated caretaking staff. This includes home nurses, general practitioners, and hospital physicians. Views for relatives are at current rather limited, but does include receiving emails or SMS based text messages.

The system is developed for the .NET platform using the $\mathrm{C \#}$ programming language for application development and ASP.NET for web development. As such, the system requires a .NET common language runtime virtual machine to run on a given platform This currently limits the system to primarily the Microsoft Windows family of PC and server operating systems, although the Mono project might LINUX enable the code with some modifications. This has not yet been tested however.

\section{FUTURE WORK}

Although a functional system has been developed and has been made available for download by others, there is still much room for improvement. Including: increased sensor support, enhanced security and higher usability. Hopefully the research community will in time help contribute to this.

Furthermore, the only valid test of the systems openness, flexibility and usefulness, will be obtained when external developers use the system for creating new assisted living prototypes and systems, providing relevant feedback.

End-user testing has not yet commenced with the system. Usability being a major design criteria, this is also of major importance, and is scheduled to commence soon.

For further details, elaborations and updates Please consult the project wiki at: http://opencareproject.com/. 


\section{REFERENCES}

[1] US Census Bureau, International Database. Accessed via Internet: http://www.census.gov/ipc/www/idb/, November 2008.

[2] T. Ezzati-Rice, D. Kashihara and S. Machlin, "Health care expenses in the United States, 2000", Agency for Helatchare Research and Quality, Report 04.0022, 2004.

[3] J.E. Bardram, A. Mihailidis, D. Wan, "Pervasive Computing in Healthcare", CRC Press, p.6, 2006

[4] "Scaling Mount Proteome to bring down chronic disease". The Pfizer Journal $\mathbb{R}$, Global Edition Volume 1I, Number 2, 2001, 4-9.R.

[5] Centers for Disease Control and Prevention, National Center for Health Statistics, Health United States, "Limittation of acitivity caused by selected chronic health conditions among working-age adults, by age United States, 2004-2005.”, 2007, Figure 16. Data from the National Health Interview Survey. Accessed via Internet: http://www.cdc.gov/nchs/data/hus/hus07.pdf, November 2008.

[6] Centers for Disease Control and Prevention, National Center for Health Statistics, Health United States, "Limittation of acitivity caused by selected chronic health conditions among older adults, by age: United States, 2004-2005.”, 2007. Data from the National Health Interview Survey. Accessed via Internet: http://www.cdc.gov/nchs/data/hus/-hus07.pdf, November 2008.

[7] J.E. Bardram, A. Mihailidis, D. Wan, "Pervasive Computing in Healthcare", CRC Press, 2006.

[8] S. Candrili, J. Mauskopf, "How much does a hospital day cost?" Presented at: $11^{\text {th }}$ Annual International Meeting of the International Society for Pharmacoeconomics and Outcomes Research May 20-24 2006. Philadelphia. PA

[9] L.M Tolstrup, A. Bonne, Dansk Regioner, Note on the Estimated Average Value of 1 Day of Hospitalization in a Danish Public Sector Hospital, data drawn from http://www.sst.dk/Planlaegning og behandling/DRG.aspx , November 2008.

[10] R. Bennefield, R. Bonnette, "Structural and occupancy characteristics of housing: 2000", United States Census Bureau, Report No. C2KBR32,2003

[11] J. Keye, T. Zitzelberger, "Overview of healthcare, disease, and disability", in "Pervasive Computing in Healthcare", CRC Press, p. 320,2006.

[12] R. Thractenberg, C. Singer, J. Kaye, "Symptoms of sleep disturbance in persons with Alzheimer's disease and normal elderly", Journal of Sleep Research 14, 177-185, 2005

[13] M. Weiser, "The computer for the 21 st century". In Scientific American, September, 1991.

[14] M. Weiser, "Some computer science issues in ubiquitous computing". In Communications of the ACM, July 36, vol. 7, 1993.

[15] D. A. Norman, "The invisible computer: why good products can fail, the personal computer is so complex, and information appliances are the solution". The MIT Press, Cambridge, Massachusetts, USA, 1998.

[16] H. Kautz, L. Arnstein, G. Borriello, O. Etzioni., D. Fox. "An overview of the assisted cognition project.", AAAI-2002 Workshop on Automation as Caregiver: The Role of IntelligentTechnology in Elder Care, Edmonton, Alberta, 2002

[17] Seon-Woo Lee, Yong-Joong Kim, Gi-Sup Lee, Byung-Ok Cho, NamHa Lee, "A remote behavioral monitoring system for elders living alone", Control, Automation and Systems, 2007. ICCAS '07. International Conference on, pages 2725_2730, Oct. 2007.

[18] C.Y. Huang and J.L. Su, "A middleware of dicom and web service for home-based elder healthcare information system". Information Technology Applications in Biomedicine, 2007. ITAB 2007. 6th International Special Topic Conference on, p. 182-185, Nov. 2007.

[19] DICOM. Accessed via Internet http://medical.nema.org/, Nov. 2008
[20] NEMA. Accessed via Internet http://www.medicalimaging.org/. November 2008

[21] U. Varshney, "Pervasive healthcare and wireless health monitoring", Mobile Networking Applications, 12(2-3)113-127, 2007

[22] A. Bamis, D. Lymberopoulos, T. Teixeira, A. Savvides, "Towards precision monitoring of elders for providing assistive services". In PETRA '08: Proceedings of the 1st international conference on Pervasive Technologies Related to Assistive Environments, pages 18, New York, NY, USA, 2008. ACM.

[23] D. Kirovski, N. Oliver, M. Sinclair, D. Tan. "Health-os: a position paper". In HealthNet '07: Proceedings of the 1st ACM SIGMOBILE international workshop on Systems and networking support for healthcare and assisted living environments, pages 76-78, New York, NY, USA, 2007. ACM.

[24] L. Schwiebert, S.K.S. Gupta, and J. Weinmann, "Research challenges in wireless networks of biomedical sensors". In MobiCom '01: Proceedings of the 7th annual international conference on Mobile computing and networking, pages 151-165, New York, NY, USA, 2001. ACM.

[25] M. Rodrig, A. LaMarca. "Oasis: an architecture for simplified data management and disconnected operation". Personal Ubiquitous Computing, 9(2):108-121, 2005.

[26] J. E. Bardram, C. Bossen, A. Thomsen, "Designing for transformations in collaboration: a study of the deployment of homecare technology." In GROUP '05: Proceedings of the 2005 international ACM SIGGROUP conference on Supporting group work, pages 294-303, New York, NY, USA, 2005. ACM.

[27] Telcomed, "About us", Accessed via Internet: http://www.telcomed.ie/about_us.html, November 2008

[28] Corscience, "Telemedicine", Accessed via Internet: http://www.corscience.de/en/medical-engineering/productssystems/telemedicine.html, November 2008

[29] Tunstall, "Telehealth Monitors", Accessed via Internet: http://www.tunstallhealthcare.com/Default.aspx?ID=2137, November 2008

[30] Intel, "Intel Health Guide Overview", Accessed via Internet: http://www.intel.com/healthcare/ps/healthguide/index.htm, November 2008

[31] Continua Alliance, "Home Page", Accessed via Internet: http://www.continuaalliance.org, November 2008.

[32] Continua Alliance, "Overview", Accessed via Internet: http://www.continuaalliance.org/about/, November 2008.

[33] Continua Alliance, "Delivering on the promise of modern medicine: the need for interopable health \& medical devices", Accessed via Internet: http://www.continuaalliance.org/news events/news/backgrounder, November 2008 .

[34] Continua Alliance, "Continua Overview Presentation", Accessed via Internet: $\quad$ http://www.continuaalliance.org/about/ContinuaOverview Presentation v10.1.pdf, November 2008.

[35] Linux Information Project Definition. "Vendor lock-in definition", Accessed via Internet: http://www.linfo.org/vendor lockin.html, September, 2007. [35]

[36] S. Wagner, "Towards an open and easily extendible home care system infrastructure", Proceedings of the 2nd International Conference on Pervasive Computing Technologies for Healthcare, Tampere, Finland 2008

[37] S. Wagner, "Zero-configuration of pervasive healthcare sensor networks" Proceedings of the The Third International Conference on Pervasive Computing and Applications (ICPCA2008), Alexandria, Egypt, 2008.

[38] R.A. Soerensen, J.M. Nygaard, "Distributed zero configuration base station", Proceedings of the $2^{\text {nd }}$ International Conference on Pervasive Computing Technologies for Healthcare, Tampere, Finland (2008). 